\title{
Progress in Japanese Telemedicine, and New Wave
}

\author{
Hiroshi Kondoh ${ }^{1,2,3 *}$ \\ ${ }^{1}$ President, Japanese Telemedicine and Tele-care Association \\ 2 Division of Medical Informatics, Tottori University Hospital, Tottori, Japan \\ ${ }^{3}$ Yonago Subcenter, Media Information Center, Tottori University, Tottori, Japan
}

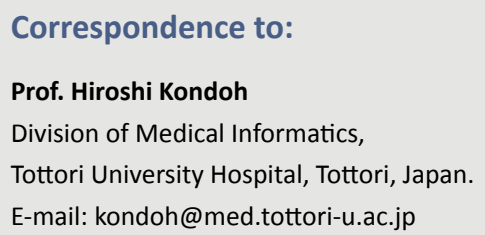

\section{Standardization}

I am a radiologist and I developed and introduced first radiology information system (RIS) in Japan in 1993 with a hard time, after the analysis of workflows. DICOM had not MWM and MPPS at that time. I developed and operated EPR and PACS sharing system using global standard XDS and XDS-I and Japanese standard SS-MIX2. The introduction took in only a year. However, it was found that standardization of contents was delayed for the first time by operation.

\section{Thin-client System}

I introduced and started the total operation of EPR in the Tottori University hospital in 2003 and it was the first time in Japanese national university hospitals. In 2008, I introduced thin-client system as an infrastructure of EPR. It was also first introduction of thin-client system as an infrastructure of EPR in Japan. In 2010, we developed and started the operation of regional EPR and PACS sharing system in Tottori prefecture, which used the thin-client system as an infrastructure and was called Oshidori-Net. In this system it used centered storage of each EPRs and PACSs with integrated patient ID because of usage thin-client infrastructure. We expected for secure system, in which clients kept no clinical data.

\section{The Introduction of Cloud Technology in EPR and PACS Sharing System}

We introduced virtual server, virtual storage, virtual network and large flash memories to Oshidori-Net in 2015. It showed faster image displaying of accessing from Heidelberg through Internet than in hospital gigabit network. It also
EJBI 2018; 14(4):03

Received: August 13, 2018

Accepted: August 14, 2018

Published: August 21, 2018 showed availability of the system, we could increase gateway servers according to increase of informant hospitals easily.

\section{Satellite Communication of} Homecare Systems Showed Needs of ASP

I had two experiences of homecare video-system with satellite communication.

First one system showed the expansion of one hospital network to patient home and university hospital. But the patient needed the communication of another hospital. So the second system was planned as an application service provider system like cloud server nowadays. The system could communicate patients and hospitals freely. The second system also transmitted patient's monitor data for early detection of respiratory infections.

\section{$5 \quad$ New Waves}

Online clinical practice has become insured from this year in Japan. This was made possible by smartphone developments. Demanded elements include the fact that video conference has become possible, information on various monitor devices and interview information can be registered in advance.

This wave connects patient's daily information to hospital electronic medical record. It seems that the remote medical treatment so far reversed the flows which the hospital system spread to the house.

Networking for acute cardiac disease and tele-stroke between staffs of hospitals were also another new wave using smartphones.

I thought that it is necessary to optimize the flows of these information. 